\title{
Offspring size and parental fitness in Daphnia magna
}

\author{
MAARTEN BOERSMA*
}

Max-Planck-Institut für Limnologie, Postfach 165, D-24302 Plön, Germany

\begin{abstract}
Summary
Variation in offspring size and number has been described for a wide range of organisms. Many theoretical models predict that in a given environment, the production of one single offspring size would yield the highest parental fitness. In most planktonic cladocerans, however, offspring size has been found to increase with size and age of the mother, and as individuals of variable size often co-occur within a population, offspring of variable sizes can be produced simultaneously. In this study, I investigated the relationship between age of the mother and size of her offspring to assess at what age of the mother the optimal offspring size was produced. Optimal offspring size was defined as that size of the offspring yielding the highest parental fitness, which translates to a definition of optimal offspring size as the one having the highest juvenile fitness per unit effort put in these juveniles. I observed that the youngest females produced offspring with the highest juvenile fitness per unit effort, and hence concluded that offspring produced by these females were of optimal size. Larger offspring produced by older females were estimated to yield only $70 \%$ of the potential fitness of optimally sized offspring.
\end{abstract}

Keywords: food; life history; predation

\section{Introduction}

One of the central themes of life-history research in the past decades has been variation in offspring size and number (see, e.g. chapters in Roff, 1992; Stearns, 1992). Generally, selection should favour the parental strategy of size and number of offspring determination that maximizes parental fitness (e.g. Lack, 1947). According to the classic paper of Smith and Fretwell (1974), maximizing parental fitness will lead to a single optimal investment per offspring for any given environment, provided that (1) a trade-off exists between size and number of individual offspring (i.e. the amount of energy invested in reproduction per breeding attempt is fixed), and (2) that as the energy expended on individual offspring increases, the fitness of individual offspring increases. The optimal investment per offspring, as proposed by Smith and Fretwell (1974), is characterized by the highest fitness of individual juveniles per unit effort. As total effort per breeding attempt does not influence the parental fitness-function, changes in total resource acquisition, or in the proportion of resources allocated to reproduction, should not change this optimal investment per offspring. Organisms that live in changing environments with respect to their food conditions may, however, use resource acquisition as an estimate of environmental circumstances which their offspring will encounter after birth. In planktonic cladocerans such as Daphnia, adults and juveniles generally show a large overlap in their resources. As a result, mothers may assess the conditions their offspring will encounter, and hence adjust the size of their offspring according to their own resource availability. This results in the often observed relationship between resource ration and egg size in cladocerans, with generally larger offspring produced at lower food levels (e.g. Tessier and Consolatti, 1991; Glazier, 1992; Guisande and Gliwicz, 1992; Ebert, 1994). In contrast to the predictions from the Smith and Fretwell model, however, it has frequently been reported that, in

*e-mail: boersma@mpil-ploen.mpg.de 
cladocerans, larger mothers produce larger offspring than smaller mothers, which results in juveniles of variable sizes being produced simultaneously in a population (Green, 1956; Gliwicz and Guisande, 1992; Ebert, 1993; Enserink et al., 1993; Lampert, 1993; Boersma, 1995). Because in cladocerans growth continues after maturity, larger females are also older.

Although many studies have dealt with optimal size and number of offspring in a wide range of organisms (see references in Roff, 1992), variation in these traits has been studied to a lesser extent (e.g. Parker and Begon, 1986; McGinley et al., 1987). Most studies on offspring size variability have focused mainly on variation in offspring size within broods. This variation is usually considered to be a bet-hedging strategy, an adaptation to variable environments (e.g. Wilbur, 1977; Kaplan, 1980; Crump, 1981; Thompson, 1984), although McGinley et al. (1987) argued that variation in offspring size is favoured only when the parents are able to direct their progeny into the appropriate habitat.

Among-brood variation in offspring size has been the subject of relatively few studies (Semlitsch, 1985; Parker and Begon, 1986; McGinley et al., 1987). In most studies concerning the relationship between maternal age and offspring size in invertebrates, the opposite relationship to that found in cladocerans has been observed; that is, older animals produce smaller offspring (Roff, 1992). This phenomenon is usually thought to be the result of depletion of resources (Roff, 1992). As amongbrood variation in offspring size is so prominent in cladocerans, a number of theories have been formulated to explain the observed dependence of offspring size on maternal size (age) in this group of animals:

1. Offspring produced by smaller animals are smaller as a result of morphological constraints. Robertson (1988) suggested that offspring size increases with maternal size in chydorids as a result of constraints such as size of the brood pouch or capacity of the ovaries. Small individuals with small brood pouches or small ovaries can only produce small eggs, a hypothesis also invoked for turtles (Congdon and Gibbons, 1987). However, as was pointed out by Glazier (1992), this hypothesis alone is not enough to explain the relationship between offspring size and maternal size, as he found that smaller females, raised at lower rations, produced eggs just as large as larger females at higher rations.

2. Glazier (1992) proposed selection for increased fecundity of young adult instars to be the driving force behind the maternal-size to offspring-size relationship. He argued that, in expanding populations, selection for increased fecundity should be stronger in younger females compared with older females. As younger females are smaller, they have smaller brood pouches, and size of the brood pouch limits the amount of egg material it can contain. Therefore, this higher selection for increased number of offspring in younger females will necessarily lead to smaller eggs being produced by these females.

3. Lampert (1993) hypothesized that the offspring-size to maternal-size relationship is an adaptation to positively size-selective predation, which is mainly exerted by fish. Predation by these positively size-selective predators will result in a smaller average adult size, which results in the production of smaller offspring, and hence animals which are smaller at maturity, and therefore less vulnerable to predation by fish. When fish predation pressure decreases, which often takes place in the second half of a growing season, daphnids will grow, and because large daphnids produce more offspring, producing offspring which are also larger will create a selective advantage. Thus younger animals are thought to produce suboptimally sized offspring to avoid fish predation.

4. If large individuals are present in the population, this indicates that predation by positively size-selective predators is probably low at that time. This will often lead to the presence of negatively size-selective predators, such as the invertebrates Chaoborus and Leptodora. This will result 
in a higher vulnerability to predation for smaller individuals, and hence a selective advantage for larger animals, producing larger offspring (Boersma, 1995; De Meester, 1995).

5. The presence of large individuals in the population implies that current fish predation is low. All else being equal, this should lead to higher densities, and hence higher levels of intraspecific competition, which will lead to a depression of resource levels. As larger offspring show greater starvation resistance (e.g. Tessier and Consolatti, 1989), large animals which produce larger eggs will have a selective advantage (Boersma, 1995; De Meester, 1995). The series of models derived by Parker and Begon (1986) predict that competition should lead to larger females laying larger eggs. Indeed, in many species, such an increase in offspring size with increasing competitive pressure has been observed (Brockelman, 1975; Christiansen and Fenchel, 1979; Sargent et al., 1987). In contrast to being large, which implies that fish predation is low at the time and hence the likelihood for intraspecific competition is high, being small (= young) does not yield any information on current predation risks and feeding conditions, as small animals will be present under all conditions. Therefore, younger females should produce offspring that are optimally adapted to the ambient food conditions at the time.

Testing the different hypotheses described above is not straightforward. However, establishing the relationship between age of the mother and fitness of young produced at the different ages might enable one to reject some of these hypotheses. If the optimal effort per offspring is achieved by large (old) mothers, then the hypotheses relating the increase in offspring size to the presence of invertebrate predators and to increased likelihood of competition can be rejected, as then an explanation is needed only as to why the offspring produced by small females are so small. Alternatively, the observation of young mothers producing optimal offspring size would allow the rejection of the morphological constraint hypothesis, the early fecundity hypothesis and the fishadaptation hypothesis, as all these hypotheses assume that optimal offspring size is produced by larger animals.

Here, I define optimal offspring size as the size of the offspring yielding highest parental fitness, which translates to a definition of optimal offspring size as the one having highest offspring fitness per unit effort (Smith and Fretwell, 1974). In cladocerans, effort per offspring is relatively easily defined. In this paper, dry weight of individual offspring was taken as a measurement of investment per offspring. Fitness is not so easily determined, as many of the fitness definitions involve measurements of contributions to the next generation (e.g. Endler, 1986), which in cyclic parthenogenetic animals such as daphnids involves some difficulties. In this study, I took the intrinsic rate of population increase, $r$, as a measurement of fitness (Stearns, 1992). Hence, for a number of adult instars of Daphnia magna, I determined the weight $(w)$ of neonates and $r$-values of successive broods, and determined fitness per unit effort, or $r / w$ for these broods. This was done for animals raised on two different food levels, in order to investigate whether the age of the mother at which optimal effort per offspring is attained is influenced by ambient food conditions.

\section{Materials and methods}

The Daphnia magna clone used in this study has been kept in the laboratory for many years, and was originally collected from a pond in Frankfurt, Germany. From a stock culture, nine juvenile animals were collected at random. These animals were placed individually in $120 \mathrm{ml}$ flow-through chambers, with a flow rate of 1 litre $\mathrm{day}^{-1}$, and fed a Scenedesmus acutus suspension with an algal carbon content of $1 \mathrm{mg}^{-1}$ at $20^{\circ} \mathrm{C}$, under continuous light conditions. The algae were grown in 3 litre chemostats in Chu-12 medium (Lampert et al., 1988). Food suspensions were prepared daily 
by resuspending centrifuged Scenedesmus in $0.45 \mu \mathrm{m}$ filtered lake water. Algal concentrations were measured spectrophotometrically.

Third brood neonates produced by this first generation of experimental animals were collected within $15 \mathrm{~h}$ of birth, and separated into two food concentrations, one high $\left(1.5 \mathrm{mg} \mathrm{C} \mathrm{l}^{-1}\right)$ and one low $\left(0.2 \mathrm{mg} \mathrm{C}^{-1}\right)$ food level, placing one animal in a flow-through chamber, with a total of nine animals per food concentration. The first food level is well above the incipient limiting level, and the second one is below this level (Lampert, 1987). From the moment these individuals matured, animals were measured and the number of offspring produced by these animals was established. To estimate the intrinsic rate of increase, $r$, of successive clutches, and to relate these measurements to the effort put into individual offspring, I established these two quantities for the first nine clutches of the experimental animals. Effort per offspring was determined by taking three neonates of each clutch, measuring their lengths and establishing their dry weights, whereas five additional neonates of the same clutch were used to establish fitness $(r)$ of the juveniles.

For the weight analysis, individual neonates were transferred to small silver weighing boats, dried for $24 \mathrm{~h}$ at $60^{\circ} \mathrm{C}$, stored in a desiccator, and weighed to the nearest $0.1 \mu \mathrm{g}$ using an electronic microbalance. Average neonate length and weight per female were then computed, and these average values per brood were used in the analyses. The intrinsic rate of increase, $r$, of each successive brood was measured by taking the five animals from these broods, and placing them together in a flow-through chamber under the same food conditions as their mothers. The $r$-values of these animals were determined using the Euler equation:

$$
\sum_{x=0}^{n} \mathrm{e}^{-r x} l_{x} m_{x}=1
$$

where $r=$ per capita rate of increase for the population $\left(\mathrm{day}^{-1}\right), x=$ age (days), $l_{x}=$ probability of surviving to age $x$, and $m_{x}=$ fecundity at age $x$. For all animals, $l_{x}$ was set to 1 ; that is, mortality observed during the experiment was assumed to be a result of handling rather than differences between individuals, as mortality in 'well-kept' cultures is normally very low (Vijverberg, 1989). The animals were cultured until they released their second brood and then discarded. Although this value of $r$, based on the release of two clutches only, is obviously an underestimation of life-time $r$, $r$-values from abbreviated life-table experiments have been shown to be highly correlated with lifetime values of $r$ (Vanni, 1986); the contribution of the first two broods reflects around $80 \%$ of total $r$ (Mooij and Boersma, 1996). Fitness per unit effort was computed by dividing observed values of $r$ by mean dry weight values per brood.

The data obtained in this experiment, based on $r$-values and mean weights of nine successive broods of nine females per food concentration, were analysed in an analysis of variance with food concentration and instar of the mother as independent variables.

\section{Results}

Food level had a significant effect on the sizes of subsequent adult instars (Fig. 1; Table 1); size at maturity (adult instar 1) was already significantly larger for animals cultured under high food conditions than for those cultured under low food conditions. Weight of individual newborns increased with age of the mothers, and neonates produced by mothers grown under low food conditions were heavier than offspring produced by mothers cultured under high food conditions (Fig. 2). The intrinsic rate of increase of offspring born from different broods also varied with food level; neonates produced at the high food level had a higher $r$-value in all cases (Fig. 3). Moreover, there was a significant increase in the value of $r$ with increasing age of the mothers for animals 


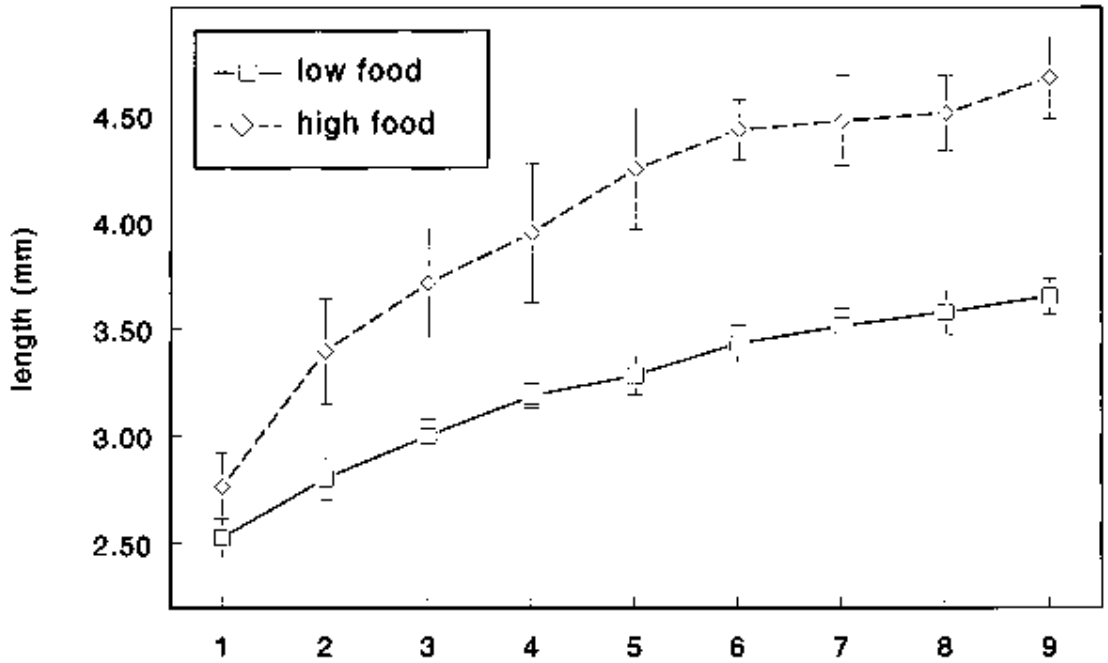

adult instar

Figure 1. Mean size $(\mathrm{mm})$ of the maternal generation at different adult instars, grown at two different food levels: high food $\left(1.5 \mathrm{mg} \mathrm{C}^{-1}\right)$ and low food $\left(0.2 \mathrm{mg} \mathrm{C}^{-1}\right)$. Error bars indicate standard deviations.

cultured under high food conditions (Pearson's correlation coefficient, $r=0.50 ; P<0.001$ ), thus implying that neonates produced by older mothers have a higher $r$-value than animals produced by younger mothers. No significant correlation of $r$-values of subsequent broods with age of the mother was found for animals cultured under low food conditions $(r=-0.04$; N.s.). Combining effort per offspring (Fig. 2) and $r$-values (Fig. 3) of the different broods, we obtain juvenile fitness per unit effort. It is important to note that the rest of the analysis depends on the assumption that a trade-off exists between size and number of offspring. This was tested by computing the partial correlation coefficient between average weight of offspring and number of offspring produced, while correcting for differences in size of the mothers (by taking mother's length and the square of

Table 1. Results of analyses of variance of the effect of food concentration and age of the mother on selected characteristics: length of the females (fem; in a repeated-measures design); weight, $r$ and $r$ /weight of the neonates ( $\mathrm{nb}$ ) (all log-transformed in the analyses); and the percentage of 'lost-fitness'

\begin{tabular}{|c|c|c|c|c|c|c|c|c|c|}
\hline \multirow[t]{2}{*}{ Characteristic } & \multicolumn{3}{|c|}{ Food level } & \multicolumn{3}{|c|}{ Instar } & \multicolumn{3}{|c|}{ Food $\times$ instar } \\
\hline & d.f. & $F$ & $P$ & d.f. & $F$ & $P$ & d.f. & $F$ & $P$ \\
\hline $\begin{array}{l}\text { Length fem } \\
(\mathrm{mm})\end{array}$ & 1,9 & 106.2 & $<0.001$ & 8,72 & 407.0 & $<0.001$ & 8,72 & 12.0 & $<0.001$ \\
\hline $\begin{array}{l}\text { Weight nb } \\
(\mu \mathrm{g})\end{array}$ & 1,132 & 79.9 & $<0.001$ & 8,132 & 31.1 & $<0.001$ & 8,132 & 2.8 & 0.007 \\
\hline$r \mathrm{nb}\left(\mathrm{day}^{-1}\right)$ & 1,114 & 766.2 & $<0.001$ & 8,114 & 2.6 & 0.012 & 8,114 & 5.3 & $<0.001$ \\
\hline $\begin{array}{l}r / \text { weight nb } \\
\left(\text { day }^{-1} \mu \mathrm{g}^{-1}\right)\end{array}$ & 1,112 & 1078.6 & $<0.001$ & 8,112 & 11.2 & $<0.001$ & 8,112 & 5.0 & $<0.001$ \\
\hline Lost-fitness & 1,112 & 0.3 & 0.969 & 8,112 & 2260.7 & $<0.001$ & 8,112 & 689.4 & $<0.001$ \\
\hline
\end{tabular}




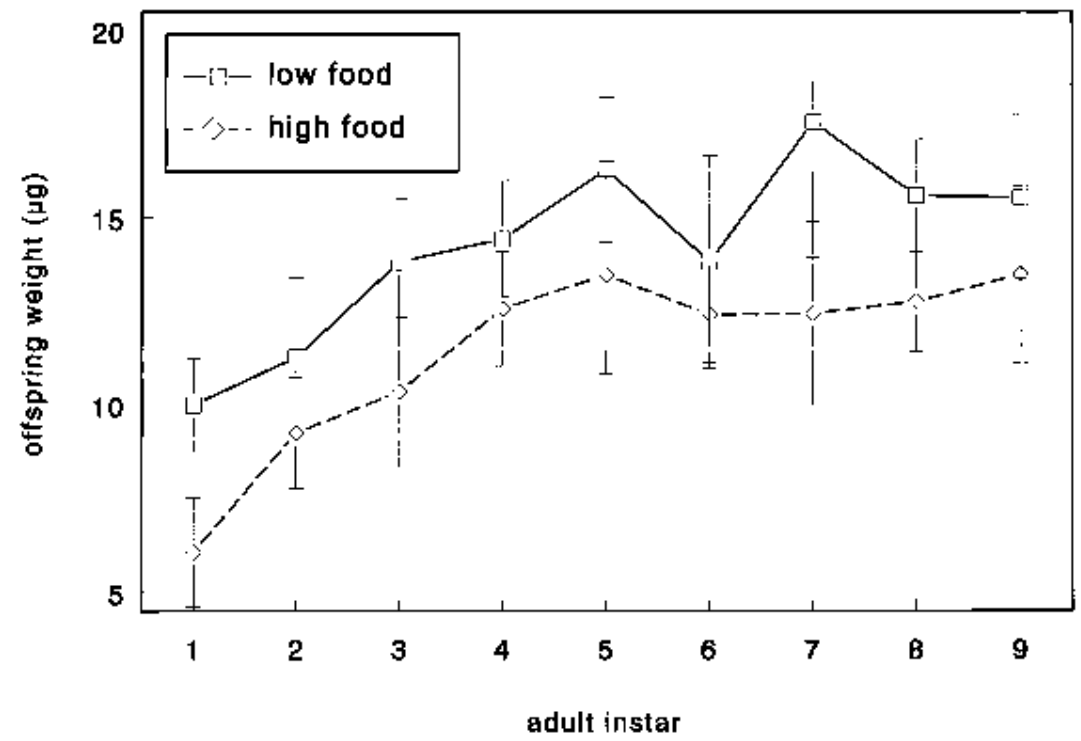

Figure 2. Mean weight $(\mu \mathrm{g})$ of neonates produced by subsequent instars of the maternal generation at two food levels. Error bars indicate standard deviations.

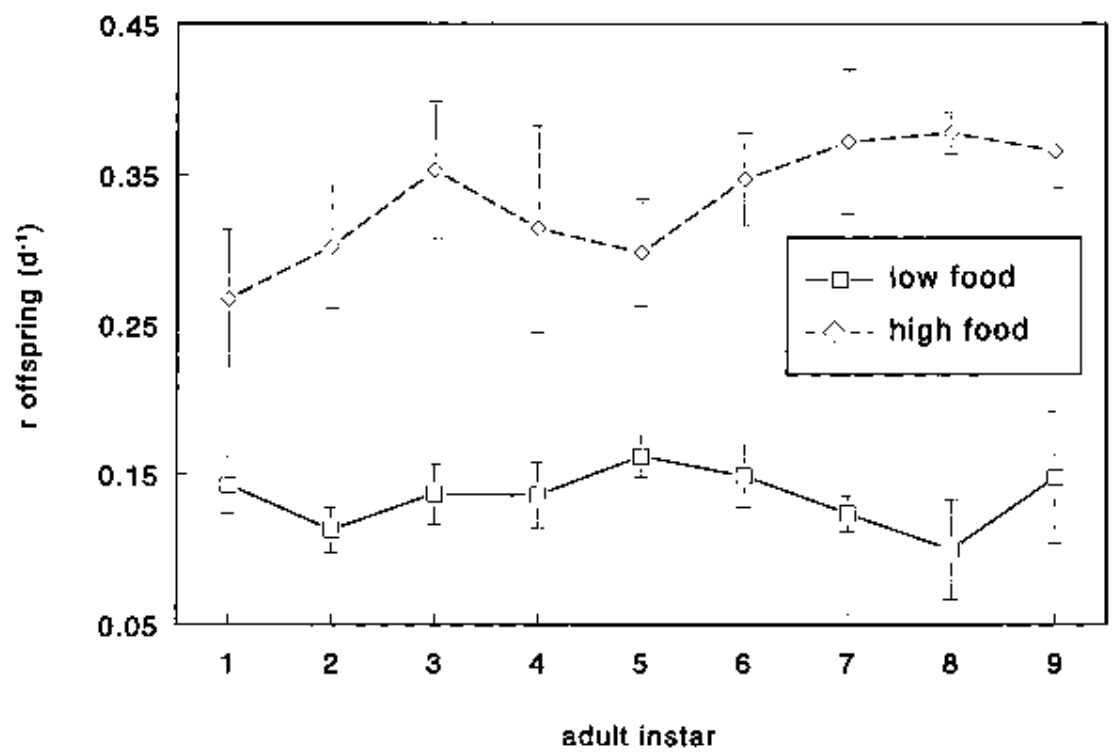

Figure 3. Mean value for the intrinsic rate of population increase, $r$, of successive broods produced by the maternal generation at two food levels. Error bars indicate standard deviations.

her length to allow deviations from a linear relationship). This yielded significantly negative partial correlation coefficients between size and number of offspring for animals under both low $(r=-0.28 ; P<0.03)$ and high $(r=-0.26 ; P<0.04)$ food conditions, thus showing the trade-off between size and number of offspring. 


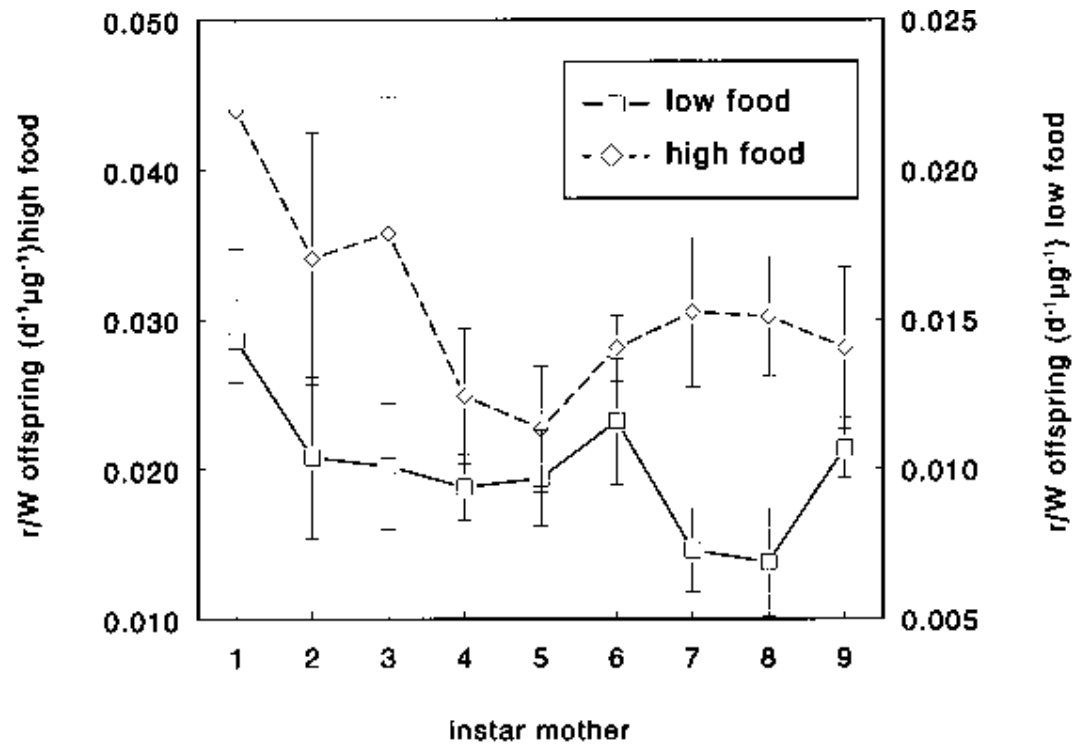

Figure 4. Fitness per unit effort of successive broods produced by the maternal generation, or the ratio between $r$ and average dry weight of successive broods at two food levels (left $y$-axis high food; right $y$-axis low food). Error bars indicate standard deviations.

Figure 4 shows the average values of fitness per unit effort for the two food levels and for different maternal instars. It is clear that neonates produced by the first adult instar have the highest fitness per unit effort for both food levels, although the absolute differences between instars are less for animals cultured under low food conditions. From this it can be concluded that the first adult instar of the Daphnia magna clone under consideration produces neonates which are optimally adapted to the environment and that, when animals get older, more energy is invested per offspring than pays off in terms of increased fitness. The effect of this 'over-feeding' of neonates on total parental fitness is rather dramatic. I estimated the percentage of 'lost fitness' for every adult instar as follows.

First the fitness, $R_{i}$, of the total brood of each adult instar, $i$, was computed by multiplying individual offspring fitness by the number of juveniles produced, or:

$$
R_{i}=r_{i} n_{i}
$$

where $r_{i}$ is the fitness per neonate and $n_{i}$ is the number of neonates. Then, from the total reproductive effort per instar (which is the product of the number of juveniles produced and their individual weight), combined with the average weight of the neonates produced by the first adult instar, $w_{1}$, the potential number of offspring all having the mass of neonates produced by the first adult instar, $n_{i}^{\prime}$, can be estimated for all subsequent adult instars:

$$
n_{i}^{\prime}=\frac{n_{i} w_{i}}{w_{1}}
$$

where $w_{i}$ is the average weight of neonates produced by the $i$ th adult instar. The product of this potential offspring number, $n_{i}^{\prime}$, and the realized fitness of first-clutch neonates, $r_{1}$, yields the po- 


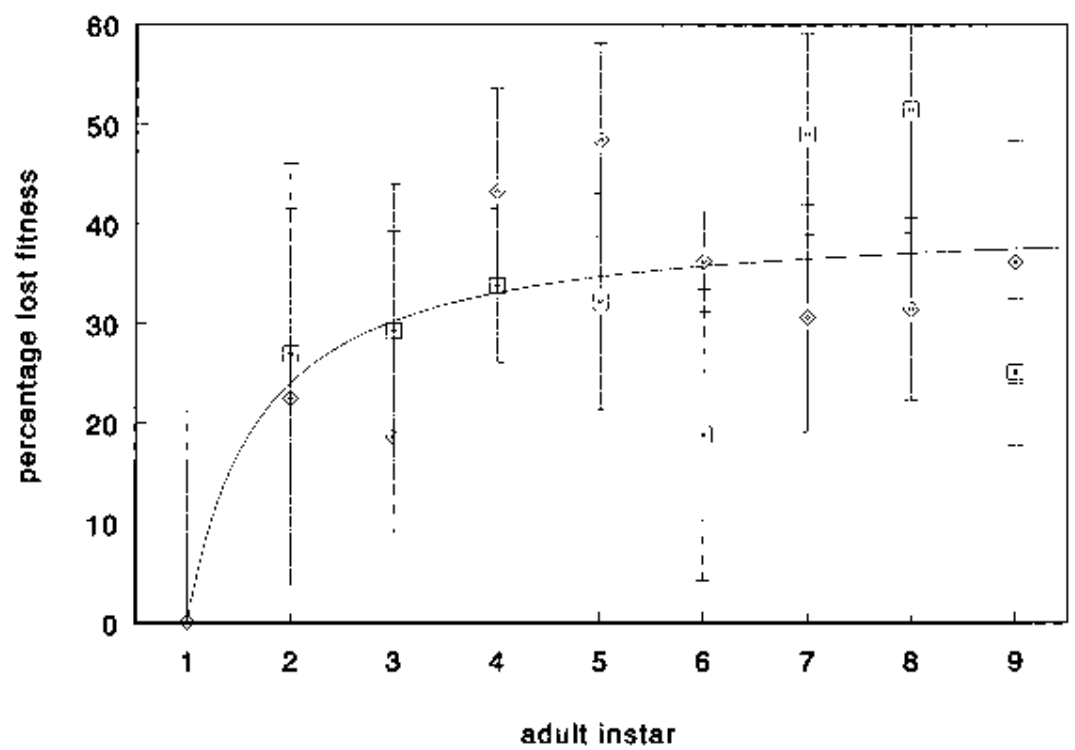

Figure 5. Percentage of the potential fitness lost by the successive broods of the maternal generation. No significant food effects were found. Error bars as in Fig. 1. A Monod-type saturation curve is fitted through the data, which explains $36 \%$ of the variance.

tential fitness contribution of each subsequent adult instar, $R_{i}^{\prime}$, if these animals produce neonates of the same weight as the ones produced by the first adult instar:

$$
R_{i}^{\prime}=n_{i}^{\prime} r_{1}
$$

Assuming that neonate weight is the only factor contributing to the between-clutch fitness differences, I expressed the fitness loss $\left(R_{i}^{\prime}-R_{i}\right)$ due to the higher neonate weight as a percentage of the potential fitness, $R_{i}^{\prime}$. Figure 5 shows the results of these computations, and reveals that the percentage of 'lost-fitness' by producing neonates which are larger than the optimal neonate size can be as high as $50 \%$, and averages $30 \%$ for adult instars $2-9$. No significant effect of food type was found on this percentage of lost fitness (Table 1).

\section{Discussion}

In this study, I have shown that effort per offspring increased with maternal age, and hence size, for Daphnia magna, both under high and low food conditions. The intrinsic rate of increase, $r$, of successive broods also increased with maternal age under high food, but not under low food, conditions. As a result of the shape of these curves, juveniles with the highest fitness per unit effort were found to be produced by primiparous individuals, both under low and high food conditions. These results suggest that parental fitness with respect to food conditions is optimized for the first brood, and that offspring produced by older females are in fact too large, when only food conditions are taken into account. Survival in natural populations of Daphnia is reported to be low, with mortality rates reaching up to $30 \%$ day $^{-1}$ for D. galeata (Vijverberg and Richter, 1982), and up to $40 \%$ day $^{-1}$ for D. magna (Lampert, 1991). A daily mortality rate of $20 \%$ would mean that with the development times observed in this study (averaging 9.8 days to release the first clutch and 3.0 days for adult instars), $10 \%$ of animals survive to release their first clutch and only $0.1 \%$ 


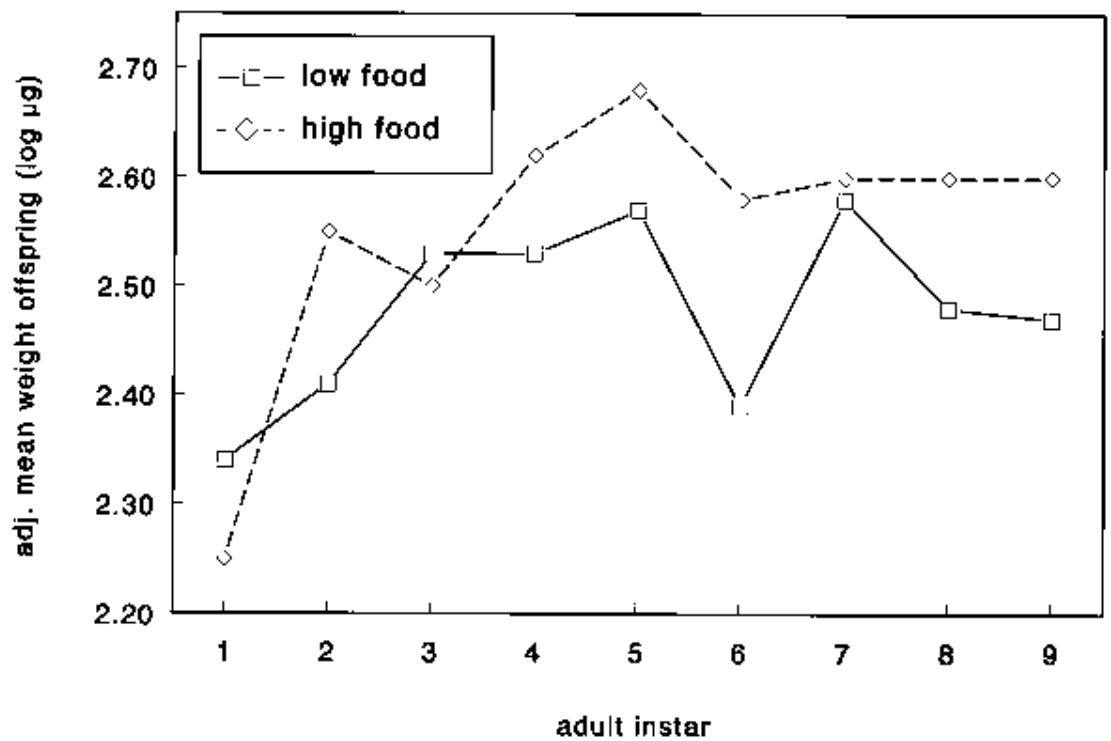

Figure 6. Adjusted mean weights resulting from an ANCOVA with food concentration and instar as independent variables, the natural logarithm of newborn weight as the dependent variable, and the natural logarithm of length as the covariable.

survive to release their third clutch. This implies that selective forces are probably much stronger for younger adult instars, as these contribute more to future generations (see Rose, 1991, for a more formal treatment of ageing and natural selection). Indeed, M. Teschner (personal communication) found genetic variability for egg size to be smaller for first broods of different D. magna clones than for later broods, suggesting higher selection for egg size in these broods.

The results described in this study, with optimally sized offspring produced by younger females, imply that three of the hypotheses relating to the dependence of offspring size on maternal size in daphnids can be rejected. The morphological constraint hypothesis (Robertson, 1988), selection for increased early fecundity (Glazier, 1992) and the hypothesis which explains the existing relationship as an adaptation to positively size-selective predation (Lampert, 1993) all assume a suboptimal offspring size for the first broods, and hence should be rejected. Only the hypothesis explaining the offspring-size to maternal-size relationship as an adaptation to negatively sizeselective predation, and the hypothesis that producing larger offspring when larger is an adaptation to possible food stress as a result of higher intraspecific competition, cannot be rejected by the data presented here. Both these hypotheses predict that offspring produced by younger individuals, again considering only current food conditions, will be more optimal in size than offspring produced by larger animals. An analysis of the length-weight relationships of neonates produced in different broods showed that the length-corrected weight increased with brood number (Fig. 6; Table 2). This implies that animals from later broods were heavier than similar sized ones from earlier broods, suggesting that the selective forces causing the relationship between maternal size and offspring size acted more on weight than on length of the offspring, which indicates that the potential food stress hypothesis is most probably correct, as invertebrate predators mainly select for length of their prey (e.g. Dodson, 1974; Spitze, 1991).

The conclusion that the relationship between maternal size and offspring size is a result of increased competition risk for larger females is in accordance with the results of Parker and Begon 
Table 2. Results of an analysis of covariance with food concentration and age of the mother as factors, the natural logarithm of neonate weight as the dependent variable, and the natural logarithm of the length of the neonates as the covariable

\begin{tabular}{lcccc}
\hline Effect & MS & d.f. & $F$ & $P$ \\
\hline Food level & 0.0237 & 1 & 1.04 & 0.309 \\
Maternal instar & 0.0692 & 8 & 3.05 & 0.004 \\
Food $\times$ instar & 0.0327 & 8 & 1.44 & 0.185 \\
Error & 0.0227 & 130 & & \\
\hline
\end{tabular}

Note: The regression slopes did not differ significantly $\left(F_{17,113}=0.85 ; P=0.64\right)$.

(1986), who used different models to predict that competition would lead to larger females producing larger eggs, but may contradict the general conclusion of McGinley et al. (1987), that variation in offspring size is favoured only when parents are able to direct their progeny in the appropriate habitat, which is difficult in aquatic environments. However, as argued here, food conditions are likely to be of inferior quality when daphnids are older, as a result of higher competition. Hence, the direction of offspring to appropriate environmental conditions seems to be carried out along a temporal axis rather than a spatial axis: environmental conditions normally change during the lifetime of a daphnid. Therefore, this does explain the apparent paradoxical observation that older animals produce offspring which are of suboptimal quality and hence that evolution has led to the production of relatively unfit attributes. Given a constant food supply, there may be a particular egg size that is optimal. With a different food supply, a different egg size would be optimal. A growing population will normally deplete its resources. Single daphnids may survive through this period of population increase, and hence throughout its life the food supply for this single daphnid is expected to decrease. I argue that this is the normal or most significant typical life-history pattern in D. magna, which leads to increases in egg size to maintain optimality throughout life. This implies that having larger eggs when older can be thought of as an appropriate response from the standpoint of lifetime fitness over the usual course of population history. The argument of Glazier (1992) and Lampert (1993) that the strategy of increasing intra-brood variation in offspring size is a much simpler bet-hedging tactic, compared to the tactic of having the fixed sequence of offspring sizes as observed in cladocerans, therefore appears incorrect (see also Philippi and Seger, 1989).

It is important to note that the analysis performed here is restricted to the analysis of the fitness of first-generation offspring. It is unclear how taking into account the number of offspring produced by second-generation animals would have affected the estimates of fitness of different broods (see also Lynch and Ennis, 1983). It is possible that as offspring of larger females are larger and hence produce a higher number of offspring, differences in fitness per unit effort as observed in this study are an overestimation. Only the culture of a number of broods of several more generations of animals would help to clarify this. Laboratory derived $r$-values as a measure of fitness do not incorporate mortality occurring in the field. Evolution of egg size will obviously have taken place with mortality present. This mortality is possibly size-specific, but this specificity is largely unknown, and likely to change within a year. Hence, it is difficult to incorporate field-mortality in computations on optimal egg size. Further studies, with different mortality scenarios, could shed light on this matter.

The existence of a trade-off between size and number of offspring produced is an essential prerequisite of the original model of Smith and Fretwell (1974), and hence also of this study. 
Because for each breeding attempt there is a fixed amount of energy available, an increasing number of offspring necessarily decreases their individual weight. Although this trade-off has been reported for many cladocerans (see a review in Ebert, 1993), it is very often blurred by the fact that different amounts of energy are allocated to reproduction, creating positive or non-significant correlations between offspring size and number (van Noordwijk and de Jong, 1986). However, correcting for differences in the size of the females, as a measure of the differences in resources allocated to reproduction, showed that there was indeed a trade-off between size and number of offspring.

In this study, I have restricted the investigations to only one clone of Daphnia magna. As the relationship between age of the mother and offspring size has been found in many cladoceran species, this trait is likely to be adaptive. To investigate whether small offspring of cladocerans always have higher fitness per unit effort values, it is necessary to repeat these experiments with a wide range of clones, originating from different habitats, and possibly including clones which produce very small offspring when they are small (e.g. the positively phototactic clones studied by De Meester, 1994). Such experiments would allow the definite rejection of some of the hypotheses proposed to explain the relationship between age of the mother and offspring size in cladocerans.

\section{Acknowledgements}

This study was supported by a grant from the European Science Foundation, and by a research fellowship from the Max-Planck Society. I thank Winfried Lampert, Hinnerk Boriss, Luc De Meester, Piet Spaak, Lawrence Slobodkin, Herwig Stibor, Martina Teschner and Larry Weider for their comments on the manuscript.

\section{References}

Boersma, M. (1995) The allocation of resources to reproduction in Daphnia galeata: Against the odds? Ecology 76, 1251-1261.

Brockelman, W.Y. (1975) Competition, the fitness of offspring, and optimal clutch size. Am. Nat. 109, 677699.

Christiansen, F.B. and Fenchel, T.M. (1979) Evolution of marine invertebrate reproductive patterns. Theor. Popul. Biol. 16, 267-282.

Congdon, J.D. and Gibbons, J.W. (1987) Morphological constraint on egg size: A challenge to optimal egg size theory? Proc. Natl. Acad. Sci. USA 84, 4145-4147.

Crump, M.L. (1981) Variation in propagule size as function of environmental uncertainty for tree frogs. $A m$. Nat. 117, 724-737.

De Meester, L. (1994) Life histories and habitat selection in Daphnia - divergent life histories of D. magna clones differing in phototactic behaviour. Oecologia 97, 333-341.

De Meester, L. (1995) Life history characteristics of Daphnia magna clones differing in phototactic behaviour. Hydrobiologia 307, 167-175.

Dodson, S.I. (1974) Adaptive change in plankton morphology in response to size-selective predation: A new hypothesis of cyclomorphosis. Limnol. Oceanogr. 19, 721-729.

Ebert, D. (1993) The trade-off between offspring size and number in Daphnia magna - the influence of genetic, environmental and maternal effects. Arch. Hydrobiol. (suppl.), 90, 453-473.

Ebert, D. (1994) Fractional resource allocation into few eggs - Daphnia as an example. Ecology 75, 568-571.

Endler, J.A. (1986) Natural Selection in the Wild. Princeton University Press, Princeton, NJ.

Enserink, L., de la Haye, M. and Maas, H. (1993) Reproductive strategy of Daphnia magna-implications for chronic toxicity tests. Aquat. Toxicol. 25, 111-123.

Glazier, D.S. (1992) Effects of food, genotype, and maternal size and age on offspring investment in Daphnia magna. Ecology 73, 910-926. 
Gliwicz, Z.M. and Guisande, C. (1992) Family planning in Daphnia - resistance to starvation in offspring born to mothers grown at different food levels. Oecologia 91, 463-467.

Green, J. (1956) Growth, size and reproduction in Daphnia (Crustacea: Cladocera). Proc. Zool. Soc. Lond 126, 173-204.

Guisande, C. and Gliwicz, Z.M. (1992) Egg size and clutch size in 2 Daphnia species grown at different food levels. J. Plankton Res. 14, 997-1007.

Kaplan, R.H. (1980) The implications of ovum size variability for offspring fitness and clutch size within several populations of salamanders (Ambystoma). Evolution 34, 51-64.

Lack, D. (1947) The significance of clutch size. Ibis 89, 309-352.

Lampert, W. (1987) Feeding and nutrition in Daphnia. Mem. Ist. Ital. Idrobiol. 45, 143-192.

Lampert, W. (1991) The dynamics of Daphnia magna in a shallow lake. Verh. Int. Ver. Limnol. 24, 795-798.

Lampert, W. (1993) Phenotypic plasticity of the size at 1st reproduction in Daphnia - the importance of maternal size. Ecology 74, 1455-1466.

Lampert, W., Schmitt, R.D. and Muck, P. (1988) Vertical migration of freshwater zooplankton: Test of some hypotheses predicting a metabolic advantage. Bull. Mar. Sci. 43, 620-640.

Lynch, M. and Ennis, R. (1983) Resource availability, maternal effects, and longevity. Exp. Gerontology 18, $147-165$.

McGinley, M.A., Temme, D.H. and Geber, M.A. (1987) Parental investment in offspring in variable environments: Theoretical and empirical considerations. Am. Nat. 130, 370-398.

Mooij, W.M. and Boersma, M. (1996) OSIRIS: An object oriented simulation framework for individual based simulations. Ecol. Model. 93, 139-153.

Parker, G.A. and Begon, M. (1986) Optimal egg size and clutch size: Effects of environment and maternal phenotype. Am. Nat. 128, 573-592.

Philippi, T. and Seger, J. (1989) Hedging one's evolutionary bets, revisited. Trends Ecol. Evol. 4, 41-44.

Robertson, A.L. (1988) Life history of some species of Chydoridae (Cladocera: Crustacea). Freshwater Biol. 20, 75-84.

Roff, D.A. (1992) The Evolution of Life Histories. Chapman and Hall, New York.

Rose, M.R. (1991). Evolutionary Biology of Aging. Oxford University Press, New York.

Sargent, R.C., Taylor, P.D. and Gross, M.R. (1987) Parental care and the evolution of egg size in fishes. Am. Nat. 129, 32-46.

Semlitsch, R.D. (1985) Reproductive strategy of a facultatively paedomorphic salamander Ambystoma talpoideum. Oecologia 65, 305-313.

Smith, C.C. and Fretwell, S.D. (1974) The optimal balance between size and number of offspring. Am. Nat. 108, 499-506.

Spitze, K. (1991) Chaoborus predation and life-history evolution in Daphnia pulex - temporal pattern of population diversity, fitness, and mean life history. Evolution 45, 82-92.

Stearns, S.C. (1992) The Evolution of Life Histories. Oxford University Press, New York.

Tessier, A.J. and Consolatti, N.L. (1989) Variation in offspring size in Daphnia and consequences for individual fitness. Oikos 56, 269-276.

Tessier, A.J. and Consolatti, N.L. (1991) Resource quantity and offspring quality in Daphnia. Ecology 72, 468-478.

Thompson, J.N. (1984) Variation among individual seed masses in Lomatium grayi (Umbelliferae) under controlled conditions: Magnitude and partitioning of variance. Ecology 65, 626-631.

Vanni, M.J. (1986) Fish predation and zooplankton demography: Indirect effects. Ecology 67, 337-354.

van Noordwijk, A.J. and de Jong, G. (1986) Acquisition and allocation of resources: Their influence on variation in life history tactics. Am. Nat. 128, 137-142.

Vijverberg, J. (1989) Culture techniques for studies on the growth, development and reproduction of copepods and cladocerans under laboratory and in situ conditions: A review. Freshwater Biol. 21, 317-373.

Vijverberg, J. and Richter, A.F. (1982) Population dynamics and production of Daphnia hyalina Leydig and Daphnia cucullata Sars in Tjeukemeer. Hydrobiologia 95, 235-259.

Wilbur, H.M. (1977) Propagule size, number, and dispersion pattern in Ambystoma and Asclepias. Am. Nat. 111, 43-68. 